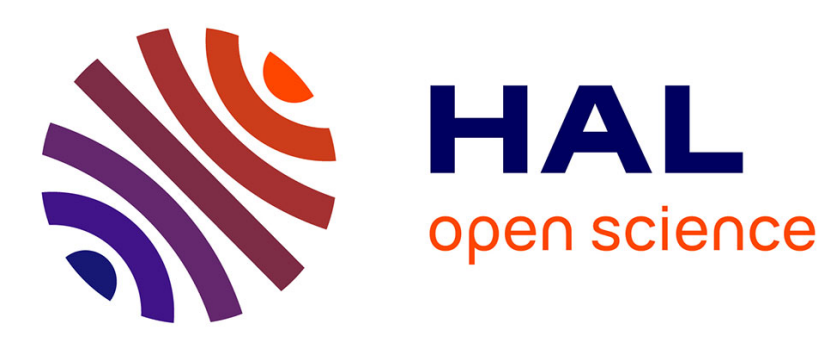

\title{
Stochastic Coalitional Better-response Dynamics and Stable Equilibrium
}

\author{
Konstantin Avrachenkov, Vikas Vikram Singh
}

\section{To cite this version:}

Konstantin Avrachenkov, Vikas Vikram Singh. Stochastic Coalitional Better-response Dynamics and

Stable Equilibrium. Mathematical Game Theory and Applications, 2016. hal-01403039

\section{HAL Id: hal-01403039 \\ https://hal.inria.fr/hal-01403039}

Submitted on 25 Nov 2016

HAL is a multi-disciplinary open access archive for the deposit and dissemination of scientific research documents, whether they are published or not. The documents may come from teaching and research institutions in France or abroad, or from public or private research centers.
L'archive ouverte pluridisciplinaire HAL, est destinée au dépôt et à la diffusion de documents scientifiques de niveau recherche, publiés ou non, émanant des établissements d'enseignement et de recherche français ou étrangers, des laboratoires publics ou privés. 


\title{
Stochastic Coalitional Better-response Dynamics and Stable Equilibrium*
}

\author{
Konstantin Avrachenkov and Vikas Vikram Singh \\ INRIA Sophia-Antipolis Mediterranee, \\ 2004 route des Lucioles, 06902 Sophia-Antipolis, France \\ E-mail: k.avrachenkov@inria.fr, vikasstar@gmail.com
}

\begin{abstract}
We consider coalition formation among players in an $n$-player finite strategic game over infinite horizon. At each time a randomly formed coalition makes a joint deviation from a current action profile such that at new action profile all the players from the coalition are strictly benefited. Such deviations define a coalitional better-response (CBR) dynamics that is in general stochastic. The CBR dynamics either converges to a $\mathcal{K}$-stable equilibrium or becomes stuck in a closed cycle. We also assume that at each time a selected coalition makes mistake in deviation with small probability that add mutations (perturbations) into CBR dynamics. We prove that all $\mathcal{K}$-stable equilibria and all action profiles from closed cycles, that have minimum stochastic potential, are stochastically stable. Similar statement holds for strict $\mathcal{K}$-stable equilibrium. We apply the CBR dynamics to study the dynamic formation of the networks in the presence of mutations. Under the CBR dynamics all strongly stable networks and closed cycles of networks are stochastically stable.
\end{abstract}

Keywords - Strong Nash equilibrium, Coalitional better-response, Stochastic stability, Network formation games, Strongly stable networks.

\section{Introduction}

Nash equilibrium is most desirable solution concept in non-cooperative game theory. When a strategic game is played repeatedly over infinite horizon then the Nash equilibrium that is played in the long run depends on an initial action profile as well as the way all the players choose their actions at each time. Several discrete time dynamics have been studied in the literature to study the Nash equilibrium selection in the long run. Young [27] considered an $n$ players strategic game where at each time all the players make a simultaneous move and each player chooses an action that is the best response to $k$ previous games among the $m, k \leq m$, most recent games in the past. In general this dynamics need not converge to a Nash equilibrium, it may stuck into a closed cycle. Young [27] also considered the case where at each time with small probability each player makes mistake and chooses some non-optimal action. These mistakes add mutations into the dynamics. In general the mutations can be sufficiently small. This leads to the definition of stability of Nash equilibrium as mutations vanish. This type of stability is called stochastic stability. Young proposed an algorithm to compute the stochastically stable Nash equilibria. For $2 \times 2$ coordination games he showed that the risk dominant Nash equilibrium is stochastically stable. Kandori et al. [18] considered a different dynamic model where at each

\footnotetext{
*The authors' names are given in alphabetic order.
} 
time each player plays with every other player in pairwise contest. The pairwise contest is given by $2 \times 2$ symmetric matrix game and each player chooses an action which has higher expected average payoff. The mutations are present into dynamics due to wrong actions taken by the players. For $2 \times 2$ coordination games they showed that a risk dominant Nash equilibrium is stochastically stable. That is, for $2 \times 2$ coordination games the dynamics given by [27] and [18] selects the same Nash equilibrium. Fudenberg et al. [10] proposed a dynamics where at each time only one player is selected to choose actions. The mutations with small probability also occur at each time. The risk dominant Nash equilibrium in $2 \times 2$ coordination games need not be stochastically stable under this dynamics.

The Nash equilibrium concept is inadequate for the situations where players can a priori communicate, being in a position to form a coalition and jointly deviate in a coordinated way. To capture such situations (strict) $\mathcal{K}$-stable equilibrium [26] is an adequate solution concept, where $\mathcal{K}$ is a set of all feasible coalitions. When there is no restriction on coalition formation, (strict) $\mathcal{K}$-stable equilibrium corresponds to (strict) strong Nash equilibrium [1]. As motivated from the application of $\mathcal{K}$-stable equilibria in network formation games considered in [7, 14, 13] we restrict ourselves to only pure actions. A $\mathcal{K}$-stable equilibrium need not always exist and in such case there exist some set of action profiles forming a closed cycle such that it is possible to reach from one action profile to another via sequence of improving deviations by the coalitions; and it is not possible to reach an action profile outside of the closed cycle from an action profile belonging to closed cycle via improving deviations by the coalitions.

Recently, some stochastic dynamics involving the coalition formation among players have been proposed [4, 21, 26]. Sawa [26] studied the stochastic stability in general finite games where the mutations are present through a logit choice rule. Newton [21] considers the situation where profitable coalitional deviations are given greater importance than unprofitable single player deviations. Avrachenkov et al. [4] considered a variant of network formation game and discuss the stochastic stability of the networks when the networks are formed dynamically, in the presence of mutations, over infinite horizon. In general, the stochastic stability results depend on the way actions being chosen during the infinite play. Some other famous works on stochastic stability in different settings include [8, 9, 10, 22, 23, 11, 25, 20, 24].

In this paper we propose a new stochastic dynamics due to the coalition formation in a strategic game over infinite horizon. We call our dynamics a coalitional better-response (CBR) dynamics where at each time players are allowed to form a coalition and make a joint deviation from the current action profile if it is strictly beneficial for all the members of the coalition. We assume that the coalition formation is random and at each time only one coalition can be formed among all feasible coalitions. We also consider the situation where at each time a selected coalition makes wrong decision with small probability, i.e., it moves to an action profile where all the players from the coalition are not strictly benefited. These mistakes work as mutations and add perturbations into CBR dynamics. We prove that the perturbed CBR dynamics selects $\mathcal{K}$-stable equilibria or closed cycles, that has minimum stochastic potential among all action profiles, in the long run as mutations vanish. If there is no restriction on coalition formation, CBR dynamics selects all the strong Nash equilibria and closed cycles, i.e., all the strong Nash equilibria and closed cycles are stochastically stable. The similar CBR dynamics can be given for the case where each time a coalition deviate from a current action profile such that all the players from the coalition are at least as well off at new action profile and at least one player is strictly better off. In this case the similar results hold for strict $\mathcal{K}$-stable equilibrium and strict strong Nash equilibrium (SSNE). Our CBR dynamics can be applied to network formation games which shows that all the strongly stable networks and closed cycles of networks are stochastically stable. Our CBR dynamics generalizes the stochastic dynamics considered in [4] by considering the general finite games. The stochastic dynamics for pairwise stable networks considered in 
[15] can also be viewed as a special case of our CBR dynamics.

The paper is organized as follows. Section 2 contains the model and few definitions. We describe the CBR dynamics in Section 3. We conclude our paper in Section 4 .

\section{The Model}

We consider an $n$-player strategic game. Let $N=\{1,2, \cdots, n\}$ be a finite set of players, and $A_{i}$ be a finite set of actions of player $i$ whose element is denoted by $a_{i}$. We denote $A=\prod_{i=1}^{n} A_{i}$ as a set of all action profiles and $a=\left(a_{1}, a_{2}, \cdots, a_{n}\right)$ denotes an element of $A$. The payoff function of player $i$ is defined as $u_{i}: A \rightarrow \mathbb{R}$. We consider settings where players may form a coalition and jointly revise their strategies. In many situations it may not be feasible to form all types of coalitions. Let $\mathcal{K} \subseteq \mathcal{P}(N) \backslash \phi$ be the set of all feasible coalitions, where $\mathcal{P}(N)$ denotes the power set of $N$ and $\phi$ an empty set. For a coalition $S \in \mathcal{K}$, define $A_{S}=\prod_{i \in S} A_{i}$ whose element is denoted by $a_{S}$ and $a_{-S}$ denotes an action profile of players outside $S$. A coalition of players jointly deviate from a current action profile if at new action profile each player from the coalition is strictly benefited. Such a deviation leads to the definition of $\mathcal{K}$-stable equilibrium. In some cases, a coalition of players jointly deviate from a current action profile if new action profile makes each player at least as well off and one player strictly better off. Such a deviation leads to the definition of strict $\mathcal{K}$-stable equilibrium.

Definition 2.1. An action profile $a^{*}$ is said to be a $\mathcal{K}$-stable equilibrium if there is no $S \in \mathcal{K}$ and $a \in A$ such that

1. $a_{i}=a_{i}^{*}, \forall i \notin S$.

2. $u_{i}(a)>u_{i}\left(a^{*}\right), \forall i \in S$.

If $\mathcal{K}=\mathcal{P}(N) \backslash \phi$, a $\mathcal{K}$-stable equilibrium is a strong Nash equilibrium (SNE) [1]. Let $A(S, a)$ be the set of all action profiles reachable from $a$ via deviation of coalition $S$. It is defined as,

$$
A(S, a)=\left\{a^{\prime} \mid a_{i}^{\prime}=a_{i}, \forall i \notin S \text { and } a_{i}^{\prime} \in A_{i}, \forall i \in S\right\} .
$$

A coalition always has option to do nothing, so $a \in A(S, a)$. Let $\mathcal{I}_{1}(S, a)$ be a set of improved action profiles reachable from an action profile $a$ via deviation of coalition $S$, i.e.,

$$
\mathcal{I}_{1}(S, a)=\left\{a^{\prime} \mid a_{i}^{\prime}=a_{i}, \forall i \notin S \text { and } u_{i}\left(a^{\prime}\right)>u_{i}(a), \forall i \in S\right\} .
$$

For an improved action profile $a^{\prime} \in \mathcal{I}_{1}(S, a)$, an action profile $a_{S}^{\prime}$ of all the players from $S$ is called a better-response of coalition $S$ against a fixed action profile $a_{-S}$ of the players outside $S$. Define, $\overline{\mathcal{I}}_{1}(S, a)=A(S, a) \backslash \mathcal{I}_{1}(S, a)$ as a set of all action profiles due to the erroneous decisions of coalition $S$. It is clear that $a \in \overline{\mathcal{I}}_{1}(S, a)$, i.e., $\overline{\mathcal{I}}_{1}(S, a)$ is always nonempty for all $S$ and $a$. A $\mathcal{K}$-stable equilibrium need not always exist. In such a case there exist a set of action profiles lying on a closed cycle and all such action profiles can be reached from each other via an improving path. The definitions of closed cycle and improving path are as follows:

Definition 2.2 (Improving Path). An improving path from a to $a^{\prime}$ is a sequence of action profiles and coalitions $a^{1}, S_{1}, a^{2}, \cdots, a^{m-1}, S_{m-1}, a^{m}$ such that $a^{1}=a, a^{m}=a^{\prime}$ and $a^{k+1} \in \mathcal{I}_{1}\left(S_{k}, a^{k}\right)$ for all $k=1,2, \cdots, m-1$.

Definition 2.3 (Cycles). A set of action profiles $C$ form a cycle if for any $a \in C$ and $a^{\prime} \in C$ there exists an improving path connecting $a$ and $a^{\prime}$. A cycle is said to be a closed cycle if no action profile in $C$ lies on an improving path leading to an action profile that is not in $C$. 
Theorem 2.4. There always exists a $\mathcal{K}$-stable equilibrium or a closed cycle of action profiles, or both $\mathcal{K}$-stable equilibrium and closed cycle.

Proof. An action profile is a $\mathcal{K}$-stable equilibrium if and only if it is not possible for any feasible coalition from set $\mathcal{K}$ to make an improving deviation from it to another action profile. So, start at an action profile. Either it is $\mathcal{K}$-stable equilibrium or there exists a coalition that can make an improving deviation to another action profile. In the first case result is established. For the second case the same thing holds, i.e., either this new action profile is a $\mathcal{K}$-stable equilibrium or there exists a coalition that can make an improving deviation to another action profile. Given the finite number of action profiles, the above process either finds an action profile which is a $\mathcal{K}$-stable equilibrium or it reaches to one of previous profiles, i.e., there exists a cycle. Thus, we have proved that there always exists either a $\mathcal{K}$-stable equilibrium or a cycle. Suppose there are no $\mathcal{K}$-stable equilibria. Given the finite number of action profiles and non-existence of $\mathcal{K}$-stable equilibria there must exists a maximal set $C$ of action profiles such that for any $a \in C$ and $a^{\prime} \in C$ there exists an improving path connecting $a$ and $a^{\prime}$, and no action profile in $C$ lies on an improving path leading to an action profile that is not in $C$. Such a set $C$ is a closed cycle.

An strict $\mathcal{K}$-stable equilibrium can be defined similarly. An action profile $a^{*}$ in Definition 2.1 is said to be strict $\mathcal{K}$-stable equilibrium if the condition 1 is same and the condition 2 is $u_{i}(a) \geq$ $u_{i}\left(a^{*}\right)$ for all $i \in S$ with at least one strict inequality. Further if $\mathcal{K}=\mathcal{P}(N) \backslash \phi, a^{*}$ is a strict strong Nash equilibrium (SSNE). In this case, for a given action profile $a$ and a coalition $S \in \mathcal{K}$ the set of improved action profiles $\mathcal{I}_{2}(S, a)$ is defined as,

$$
\mathcal{I}_{2}(S, a)=\left\{a^{\prime} \mid a_{i}^{\prime}=a_{i}, \forall i \notin S, \text { and } u_{i}\left(a^{\prime}\right) \geq u_{i}(a), \forall i \in S, u_{j}\left(a^{\prime}\right)>u_{j}(a) \text {, for some } j \in S\right\} \text {. }
$$

and $\overline{\mathcal{I}}_{2}(S, a)=A(S, a) \backslash \mathcal{I}_{2}(S, a)$. The definitions of improving path and cycles can be defined analogously to previous case. A result similar to Theorem 2.4 holds, i.e., there always exists at least a strict $\mathcal{K}$-stable equilibrium or a closed cycle of action profiles or both. A strict $\mathcal{K}$-stable equilibrium is always a $\mathcal{K}$-stable equilibrium, i.e., the set of strict $\mathcal{K}$-stable equilibrium is a subset of the set of $\mathcal{K}$-stable equilibrium.

Now, we give few examples of two player game illustrating the presence of $\mathcal{K}$-stable equilibrium and closed cycle. In particular, we allow only the coalitions of size 1 , and hence a $\mathcal{K}$-stable equilibrium is a Nash equilibrium.

Example 2.5. Consider a two player game

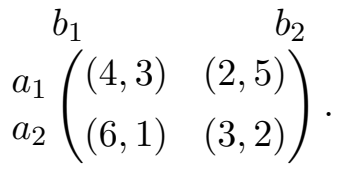

Here $\left(a_{2}, b_{2}\right)$ is the only Nash equilibrium that can be reached from all other action profiles via improving deviations. The situation is described in Figure 1.

A directed edge $\left(a_{1}, b_{1}\right) \stackrel{\{1\}}{\longrightarrow}\left(a_{2}, b_{1}\right)$ of Figure 1 represents a deviation by player 1 . The other directed edges are similarly defined. The self loop at $\left(a_{2}, b_{2}\right)$ shows that a unilateral deviation from $\left(a_{2}, b_{2}\right)$ is not possible.

Example 2.6. Consider a two player game

$$
\begin{array}{ccc}
b_{1} & b_{2} & b_{3} \\
a_{1} \\
a_{2} \\
a_{3}
\end{array}\left(\begin{array}{ccc}
(4,4) & (0,0) & (0,0) \\
(0,0) & (4,5) & (1,6) \\
(0,0) & (2,5) & (6,1)
\end{array}\right) .
$$




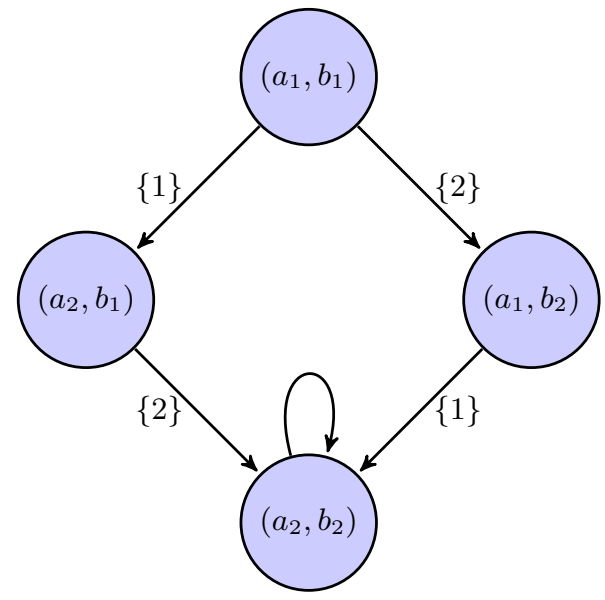

Figure 1: Nash equilibrium and Improving deviations

The Example 2.6 has both Nash equilibrium and closed cycle. The action profile $\left(a_{1}, b_{1}\right)$ is a Nash equilibrium and the closed cycle is given by Figure 2 .

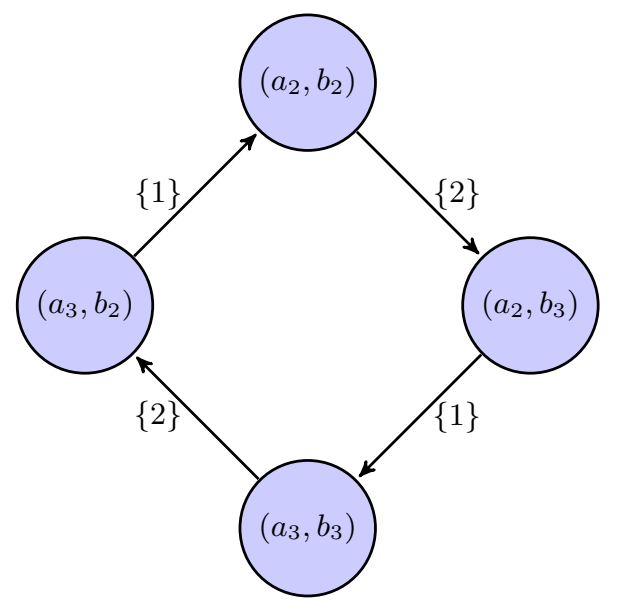

Figure 2: Closed Cycle

\section{Dynamic play}

We consider the situation where $n$ players play the strategic game, defined in Section 2 , We assume that the players can a priori communicate with each other and hence they can form a coalition. Then, they jointly deviate from the current action profile to a new action profile if new action profile is strictly beneficial for all the members of the coalition. We consider the coalition formation over infinite horizon. At each time a coalition is randomly formed and it makes an improving deviation from current action profile to a new action profile according to the improved action profile sets defined by (1). That is, at new action profile the actions of the players outside the coalition remain same as before and each player of the coalition is strictly benefited. If there is no such improved action profile for a coalition then it does not deviate. The same thing repeats at next stage and it continues for infinite horizon. Such deviations define a coalitional 
better-response (CBR) dynamics. We assume that the coalition formation is random and at each time only one coalition can be formed. If there are more than one improved action profiles for a coalition then each improved action profile can be chosen with positive probability. That is, the CBR dynamics is stochastic. The CBR dynamics defines a Markov chain over a finite set of action profiles $A$. We also assume that at each time a selected coalition makes mistake and make a joint deviation to an action profile where all the members of selected coalition are not strictly benefited. This happens with very small probability. Such mistakes add mutations into CBR dynamics. These mutations add another level of stochasticity in the CBR dynamics and as a result we have perturbed Markov chain, see e.g., 2, 3. We are interested in the action profile which is going to be selected by the CBR dynamics as mutations vanish. We next describe the stochastic CBR dynamics as discussed above.

\subsection{A stochastic CBR dynamics without mistakes}

At each time $t=0,1,2, \cdots$ a coalition $S_{t}$ is selected randomly with probability $p_{S_{t}}>0$. We assume that at each time selected coalition makes an improving deviation from current action profile $a^{t}$, i.e., at time $t+1$, the new action profile is $a^{t+1} \in \mathcal{I}_{1}\left(S_{t}, a^{t}\right)$ with probability $p_{\mathcal{I}_{1}}\left(a^{t+1} \mid S_{t}, a^{t}\right)$ where $p_{\mathcal{I}_{1}}\left(\cdot \mid S_{t}, a^{t}\right)$ is a probability distribution over finite set $\mathcal{I}_{1}\left(S_{t}, a^{t}\right)$. If there is no improving deviation for coalition $S_{t}, a^{t+1}=a^{t}$. Let $X_{t}^{0}$ denotes the action profile at time $t$, then $\left\{X_{t}^{0}\right\}_{t=0}^{\infty}$ is a finite Markov chain on set $A$. The transition law $P^{0}$ of the Markov chain is defined as follows:

$$
P^{0}\left(X_{t+1}^{0}=a^{\prime} \mid X_{t}^{0}=a\right)=\sum_{S \in \mathcal{K} ; \mathcal{I}_{1}(S, a) \neq \phi} p_{S} p_{\mathcal{I}_{1}}\left(a^{\prime} \mid S, a\right) 1_{\mathcal{I}_{1}(S, a)}\left(a^{\prime}\right)+\sum_{S \in \mathcal{K} ; \mathcal{I}_{1}(S, a)=\phi} p_{S} 1_{\left\{a^{\prime}=a\right\}}\left(a^{\prime}\right),
$$

where $1_{B}$ is an indicator function for a given set $B$. It is clear that the $\mathcal{K}$-stable equilibria and closed cycles are the recurrent classes of $P^{0}$. A $\mathcal{K}$-stable equilibrium corresponds to an absorbing state of $P^{0}$ and a closed cycle corresponds to a recurrent class of $P^{0}$ having more than one action profiles.

From Example 2.6 it is clear that in general the closed cycles together with $\mathcal{K}$-stable equilibria can be present in a game. In that case the CBR dynamics need not converge. In Example 2.6 the CBR dynamics need not converge to Nash equilibrium $\left(a_{1}, b_{1}\right)$ because once CBR dynamics enter into closed cycle given in Figure 2 then it will never come out of it. The closed cycle $C=\left\{\left(a_{2}, b_{2}\right),\left(a_{2}, b_{3}\right),\left(a_{3}, b_{3}\right),\left(a_{3}, b_{2}\right)\right\}$ is a recurrent class and $\left(a_{1}, b_{1}\right)$ is an absorbing state of Markov chain $P^{0}$ corresponding to the game given in Example 2.6.

We call a game acyclic if it has no closed cycles. The acyclic games include coordination games. There exists at least one $\mathcal{K}$-stable equilibrium for acyclic games from Theorem 2.4. For acyclic games the Markov chain defined by (3) is absorbing. Hence, from the theory of Markov chain the CBR dynamics given in Section 3.1 will be at $\mathcal{K}$-stable equilibrium in the long run no matter from where it starts (see [19]).

\subsection{A stochastic CBR dynamics with mistakes}

Now, we assume that at each time $t$ a selected coalition $S_{t}$ makes error in making a deviation from $a^{t}$ and as a result it moves to an action profile where some players in the coalition $S_{t}$ are not strictly better off. We assume that at action profile $a^{t}$, coalition $S_{t}$ makes error with probability $\varepsilon f\left(S_{t}, a^{t}\right) \in(0,1)$, where $f\left(S_{t}, a^{t}\right)$ takes into account the fact that some coalitions can be more prone to make errors than others and that some action profiles may lead to wrong choices more often than others. The parameter $\varepsilon$ allows us to tune the frequency of errors. Therefore, at 
time $t+1$ the coalition $S_{t}$ selects an improving deviation with probability $\left(1-\varepsilon f\left(S_{t}, a^{t}\right)\right)$. In particular it selects $a^{t+1} \in \mathcal{I}_{1}\left(S_{t}, a^{t}\right)$ according to distribution $p_{\mathcal{I}_{1}}(\cdot)$ defined above. By combining the probabilities we obtain that the coalition selects $a^{t+1} \in \mathcal{I}_{1}\left(S_{t}, a^{t}\right)$ with probability $\left(1-\varepsilon f\left(S_{t}, a^{t}\right)\right) p_{\mathcal{I}_{1}}\left(a^{t+1} \mid S_{t}, a^{t}\right)$. The coalition selects a non-improving deviation with probability $\varepsilon f\left(S_{t}, a^{t}\right)$. Let $p_{\overline{\mathcal{I}}_{1}}\left(\cdot \mid S_{t}, a^{t}\right)$ be a probability distribution over finite set $\overline{\mathcal{I}}_{1}\left(S_{t}, a^{t}\right)$, the coalition chooses $a^{t+1} \in \overline{\mathcal{I}}_{1}\left(S_{t}, a^{t}\right)$ with probability $\varepsilon f\left(S_{t}, a^{t}\right) p_{\overline{\mathcal{I}}_{1}}\left(\cdot \mid S_{t}, a^{t}\right)$. If there is no improving deviation, then with probability $\left(1-\varepsilon f\left(S_{t}, a^{t}\right)\right)$ the coalition does not modify the action profile, i.e., $a^{t+1}=a^{t}$, and with the complementary probability selects an action profile in $\overline{\mathcal{I}}_{1}\left(S_{t}, a^{t}\right)$ according to the distribution $p_{\overline{\mathcal{I}}_{1}}\left(\cdot \mid S_{t}, a^{t}\right)$. The transition law $P^{\varepsilon}$ of perturbed Markov chain $\left\{X_{t}^{\varepsilon}\right\}_{t=0}^{\infty}$ is defined as below:

$$
\begin{array}{r}
P^{\varepsilon}\left(X_{t+1}^{\varepsilon}=a^{\prime} \mid X_{t+1}^{\varepsilon}=a\right)=\sum_{S \in \mathcal{K} ; \mathcal{I}_{1}(S, a) \neq \phi} p_{S}\left((1-\varepsilon f(S, a)) p_{\mathcal{I}_{1}}\left(a^{\prime} \mid S, a\right) 1_{\mathcal{I}_{1}(S, a)}\left(a^{\prime}\right)\right. \\
\left.+\varepsilon f(S, a) p_{\overline{\mathcal{I}}_{1}}\left(a^{\prime} \mid S, a\right) 1_{\overline{\mathcal{I}}_{1}(S, a)}\left(a^{\prime}\right)\right) \\
+\sum_{S \in \mathcal{K} ; \mathcal{I}_{1}(S, a)=\phi} p_{S}\left((1-\varepsilon f(S, a)) 1_{\left\{a^{\prime}=a\right\}}\left(a^{\prime}\right)\right. \\
\left.+\varepsilon f(S, a) p_{\overline{\mathcal{I}}_{1}}\left(a^{\prime} \mid S, a\right) 1_{\overline{\mathcal{I}}_{1}(S, a)}\left(a^{\prime}\right)\right),
\end{array}
$$

for all $a, a^{\prime} \in A$.

The perturbed Markov chain $\left\{X_{t}^{\varepsilon}\right\}_{t=0}^{\infty}$ is irreducible because given nonzero errors it is possible to reach all the action profiles starting from any action profile in a finite number of steps. It is also aperiodic because with positive probability the state does not change. Hence, there exists a unique stationary distribution $\mu^{\varepsilon}$ for perturbed Markov chain. However, when $\varepsilon=0$, there can be several stationary distributions corresponding to different $\mathcal{K}$-stable equilibria or closed cycles. Such Markov chains are called singularly perturbed Markov chains, 2, 3]. We are interested in the action profiles to which stationary distribution $\mu^{\varepsilon}$ assigns positive probability as $\varepsilon \rightarrow 0$. This leads to the definition of a stochastically stable action profile.

Definition 3.1. An action profile $a$ is stochastically stable relative to process $P^{\varepsilon}$ if $\lim _{\varepsilon \rightarrow 0} \mu_{a}^{\varepsilon}>0$.

We recall few definitions from Young [27]. If $P^{\varepsilon}\left(a^{\prime} \mid a\right)>0, a, a^{\prime} \in A$, the one step resistance from an action profile $a$ to an action profile $a^{\prime} \neq a$ is defined as the minimum number of mistakes (mutations) that are required for the transition from $a$ to $a^{\prime} \neq a$ and it is denoted by $r\left(a, a^{\prime}\right)$. From (4) it is clear that the transition from $a$ to $a^{\prime}$ has the probability of order $\varepsilon$ if $a^{\prime} \notin \mathcal{I}_{1}(S, a)$ for all $S$ and thus has resistance 1 and is of order 1 otherwise, so has resistance 0 . So, in our setting $r\left(a, a^{\prime}\right) \in\{0,1\}$ for all $a, a^{\prime} \in A$. A zero resistance between two action profiles corresponds to a transition with positive probability under $P^{0}$. One can view the action profiles as the nodes of a directed graph that has no self loops and the weight of a directed edge between two different nodes is represented by one step resistance between them. Since $P^{\varepsilon}$ is an irreducible Markov chain then there must exist at least one directed path between any two recurrent classes $H_{i}$ and $H_{j}$ of $P^{0}$ which starts from $H_{i}$ and ends at $H_{j}$. The resistance of any path is defined as the sum of the weights of the corresponding edges. The resistance of a path which is minimum among all paths from $H_{i}$ to $H_{j}$ is called as resistance from $H_{i}$ to $H_{j}$ and it is denoted by $r_{i j}$. The resistance from any action profile $a^{i} \in H_{i}$ to any action profile $a^{j} \in H_{j}$ is $r_{i j}$ because inside $H_{i}$ and $H_{j}$ action profiles are connected with a path of zero resistance. Now we recall the definition of stochastic potential of a recurrent class $H_{i}$ of $P^{0}$ from [27]. It can 
be computed by restricting to a reduced graph. Construct a graph $\mathcal{G}$ where total number of nodes are the number of recurrent classes of $P^{0}$ (one action profile from each recurrent class) and a directed edge from $a^{i}$ to $a^{j}$ is weighted by $r_{i j}$. Take a node $a^{i} \in \mathcal{G}$ and consider all the spanning trees such that from every node $a^{j} \in \mathcal{G}, a^{j} \neq a^{i}$, there is a unique path directed from $a^{j}$ to $a^{i}$. Such spanning trees are called $a^{i}$-trees. The resistance of an $a^{i}$-tree is the sum of the resistances of its edges. The stochastic potential of $a^{i}$ is the resistance of an $a^{i}$-tree having minimum resistance among all $a^{i}$-trees. The stochastic potential of each node in $H_{i}$ is same and it is a stochastic potential of $H_{i}$ [27].

Theorem 3.2. For the stochastic CBR dynamics defined in Section 3.2, all $\mathcal{K}$-stable equilibria and all the action profiles from closed cycles, that have minimum stochastic potential, are stochastically stable. Furthermore, if one action profile in a closed cycle is stochastically stable then all the action profiles in the closed cycle are stochastically stable.

Proof. We know that the Markov chain $P^{\varepsilon}$ is aperiodic and irreducible. From (3) and (4) it is easy to see that

$$
\lim _{\varepsilon \rightarrow 0} P^{\varepsilon}\left(a^{\prime} \mid a\right)=P^{0}\left(a^{\prime} \mid a\right), \forall a, a^{\prime} \in A .
$$

From (4) it is clear that, if $P^{\varepsilon}\left(a^{\prime} \mid a\right)>0$ for some $\varepsilon \in\left(0, \varepsilon_{0}\right]$, then we have

$$
0<\varepsilon^{-r\left(a, a^{\prime}\right)} P^{\varepsilon}\left(a^{\prime} \mid a\right)<\infty .
$$

Markov chain $P^{\varepsilon}$ satisfies all three required conditions of Theorem 4 in [27] from which it follows that as $\varepsilon \rightarrow 0, \mu^{\varepsilon}$ converges to a stationary distribution $\mu^{0}$ of $P^{0}$ and an action profile $a$ is stochastically stable, i.e., $\mu_{a}^{0}>0$, if and only if $a$ is contained in a recurrent class of $P^{0}$ having minimum stochastic potential. We know that the recurrent classes of Markov chain $P^{0}$ are $\mathcal{K}$-stable equilibria or closed cycles. Therefore, all the $\mathcal{K}$-stable equilibria and the action profiles from closed cycles having minimum stochastic potential are stochastically stable. The proof of last part follows from the fact that the stochastic potential of each action profile in a closed cycle is the same.

Remark 3.3. The stochastic stability results do not depend on the function $f(\cdot)$ or the distributions of $p_{\mathcal{I}_{1}}(\cdot), p_{\overline{\mathcal{I}}_{1}}(\cdot)$ and $p=\left(p_{S}\right)_{S \in \mathcal{K}}$.

Corollary 3.4. If $\mathcal{K}=\mathcal{P}(N) \backslash \phi$, all strong Nash equilibria and all action profiles from closed cycles are stochastically stable for the stochastic CBR dynamics defined in Section 3.Q.

Proof. If $\mathcal{K}=\mathcal{P}(N) \backslash \phi$, all the strong Nash equilibria and closed cycles are the recurrent classes of $P^{0}$. Now, it is always possible to reach one action profile from another action profile by at most one error due to the formation of grand coalition. Then, the resistance $r_{i j}$ between any two distinct recurrent classes $H_{i}$ and $H_{j}$ is always 1 . Hence, the stochastic potential of each recurrent class of $P^{0}$ is $J-1$, where $J$ is the number of recurrent classes of $P^{0}$. In fact, a spanning tree in graph $\mathcal{G}$ includes only $J-1$ links and each of them has resistance 1 . The proof then follows from Theorem 3.2 .

We can have a similar CBR dynamics without mistakes and with mistakes as given in Sections 3.1 and 3.2 respectively, if for all $S \in \mathcal{K}$ and $a \in A$ the set of improved action profiles is $\mathcal{I}_{2}(S, a)$ defined by (2). We have the following results.

Theorem 3.5. For a stochastic CBR dynamics corresponding to improved action profile sets defined by (2), all strict $\mathcal{K}$-stable equilibria and all the action profiles from closed cycles, that have 
minimum stochastic potential, are stochastically stable. Furthermore, if one action profile in a closed cycle is stochastically stable then all the action profiles in the closed cycle are stochastically stable.

Proof. The proof follows from the similar arguments given in Theorem 3.2

Corollary 3.6. If $\mathcal{K}=\mathcal{P}(N) \backslash \phi$, all strict strong Nash equilibria and all action profiles from closed cycles are stochastically stable for the stochastic CBR dynamics corresponding to improved action profile sets defined by (2).

Proof. The proof follows from the similar arguments given in Corollary 3.4 .

\subsubsection{Equilibrium selection in coordination games}

First we consider a $2 \times 2$ coordination game and discuss which Nash equilibrium is selected, by CBR dynamics defined in Section 3.2 , in the long run when probability of making mistakes vanish. Our equilibrium selection results in $2 \times 2$ coordination games are different from the results given in [18], 27]. We first consider the case where only the coalitions of size 1 are formed. In this case, a $\mathcal{K}$-stable equilibrium is a Nash equilibrium. Consider a $2 \times 2$ coordination game,

$$
s_{1}\left(\begin{array}{cc}
s_{1} & s_{2} \\
s_{2}\left(a_{11}, b_{11}\right) & \left(a_{12}, b_{12}\right) \\
\left(a_{21}, b_{21}\right) & \left(a_{22}, b_{22}\right)
\end{array}\right),
$$

where $a_{j k}, b_{j k} \in \mathbb{R}, j, k \in\{1,2\}$ and $a_{11}>a_{21}, b_{11}>b_{12}, a_{22}>a_{12}, b_{22}>b_{21} . A_{i}=\left\{s_{1}, s_{2}\right\}$, $i=1,2$. Here $\left(s_{1}, s_{1}\right)$ and $\left(s_{2}, s_{2}\right)$ are two Nash equilibria. In this game there are two types of Nash equilibria one is payoff dominant and other one is risk dominant. If $a_{11}>a_{22}, b_{11}>b_{22}$, then $\left(s_{1}, s_{1}\right)$ is payoff dominant and if $a_{11}<a_{22}, b_{11}<b_{22}$, then $\left(s_{2}, s_{2}\right)$ is payoff dominant. In other cases payoff dominant Nash equilibrium does not exist. From [27, define,

$$
\begin{aligned}
& R_{1}=\min \left\{\frac{a_{11}-a_{21}}{a_{11}-a_{12}-a_{21}+a_{22}}, \frac{b_{11}-b_{12}}{b_{11}-b_{12}-b_{21}+b_{22}}\right\}, \\
& R_{2}=\min \left\{\frac{a_{22}-a_{12}}{a_{11}-a_{12}-a_{21}+a_{22}}, \frac{b_{22}-b_{21}}{b_{11}-b_{12}-b_{21}+b_{22}}\right\} .
\end{aligned}
$$

If $R_{1}>R_{2}$, then $\left(s_{1}, s_{1}\right)$ is risk dominant Nash equilibrium and if $R_{2}>R_{1}$, then $\left(s_{2}, s_{2}\right)$ is risk dominant Nash equilibrium.

The state space of Markov chain is $\left\{\left(s_{1}, s_{1}\right),\left(s_{1}, s_{2}\right),\left(s_{2}, s_{1}\right),\left(s_{2}, s_{2}\right)\right\}$, where $\left(s_{1}, s_{1}\right)$ and $\left(s_{2}, s_{2}\right)$ are the absorbing states of Markov chain $P^{0}$. From Remark 3.3 , the stochastic stability results do not depend on the distributions of $p_{\mathcal{I}_{1}}(\cdot), p_{\overline{\mathcal{I}}_{1}}(\cdot)$ and $p=\left(p_{S}\right)_{S \in \mathcal{K}}$ and function $f(\cdot)$. Therefore, we assume that all the distributions are uniform and function $f(\cdot)$ has constant value 1 . Under this assumption, the transition probability matrix of perturbed Markov chain is

$$
P^{\varepsilon}=\left(\begin{array}{cccc}
1-\frac{\varepsilon}{2} & \frac{\varepsilon}{4} & \frac{\varepsilon}{4} & 0 \\
\frac{1-\varepsilon}{2} & \varepsilon & 0 & \frac{1-\varepsilon}{2} \\
\frac{1-\varepsilon}{2} & 0 & \varepsilon & \frac{1-\varepsilon}{2} \\
0 & \frac{\varepsilon}{4} & \frac{\varepsilon}{4} & 1-\frac{\varepsilon}{2}
\end{array}\right) .
$$

The unique stationary distribution of $P^{\varepsilon}$ is $\mu^{\varepsilon}=\left(\frac{1-\varepsilon}{2-\varepsilon}, \frac{\varepsilon}{2(2-\varepsilon)}, \frac{\varepsilon}{2(2-\varepsilon)}, \frac{1-\varepsilon}{2-\varepsilon}\right)$. As $\varepsilon \rightarrow 0$, $\mu^{\varepsilon} \rightarrow\left(\frac{1}{2}, 0,0, \frac{1}{2}\right)$. That is, both the Nash equilibria are stochastically stable. This happens 
because we require only 1 mutation to reach from one Nash equilibrium to another Nash equilibrium. Therefore, the resistance from one Nash equilibrium to another Nash equilibrium is 1. Then, the stochastic potential of both the Nash equilibria will be 1. For the case when all types of coalitions can be formed the CBR dynamics always selects a payoff dominant Nash equilibrium whenever it exists because it is an SNE. When payoff dominant Nash equilibrium does not exist then both the Nash equilibria are strong Nash equilibria and in that case CBR dynamics selects both the Nash equilibria. The stochastic dynamics by Young [27] and Kandori et al. [18] always select a risk dominant Nash equilibrium.

Among symmetric coordination games if we go beyond $2 \times 2$ matrix games the result by Young [27] cannot be generalized, i.e., it need not select a risk dominant Nash equilibrium. Consider an example of $3 \times 3$ matrix game from [27,

\begin{tabular}{|c|c|c|c|}
\hline & $s_{1}$ & $s_{2}$ & $s_{3}$ \\
\hline & $(6,6)$ & $(0,5)$ & $(0,0)$ \\
\hline & $(5,0)$ & $(7,7)$ & $(5,5)$ \\
\hline & $(0,0)$ & $(5,5)$ & $(8,8))$ \\
\hline
\end{tabular}

Here $\left(s_{1}, s_{1}\right),\left(s_{2}, s_{2}\right)$ and $\left(s_{3}, s_{3}\right)$ are three Nash equilibria. The stochastic dynamics by Young [27] selects $\left(s_{2}, s_{2}\right)$ that is not a risk dominant Nash equilibrium. A Nash equilibrium of an $m \times m$ symmetric coordination game is risk dominant if it is risk dominant in all pairwise contest (see [12]). We now discuss the equilibrium selection by CBR dynamics in above $3 \times 3$ coordination game. We first consider the case where only the coalitions of size 1 are formed. The state space of Markov chain is $\left\{\left(s_{1}, s_{1}\right),\left(s_{1}, s_{2}\right),\left(s_{1}, s_{3}\right),\left(s_{2}, s_{1}\right),\left(s_{2}, s_{2}\right),\left(s_{2}, s_{3}\right),\left(s_{3}, s_{1}\right),\left(s_{3}, s_{2}\right),\left(s_{3}, s_{3}\right)\right\}$, where $\left(s_{1}, s_{1}\right),\left(s_{2}, s_{2}\right)$, and $\left(s_{3}, s_{3}\right)$ are absorbing states of Markov chain $P^{0}$. We label the states $\left(s_{1}, s_{1}\right)$ as $1,\left(s_{2}, s_{2}\right)$ as 2 and $\left(s_{3}, s_{3}\right)$ as 3 . Then, the resistance from $\left(s_{1}, s_{1}\right)$ to $\left(s_{2}, s_{2}\right)$ is denoted by $r_{12}$, where $r_{12}=1$. Similarly, $r_{13}=2, r_{31}=2, r_{21}=1, r_{23}=1, r_{32}=1$. There are three 1-trees as given below.
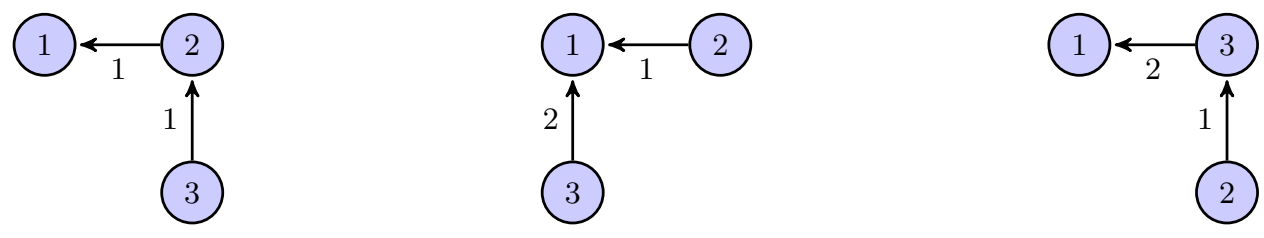

Figure 3: 1-trees

The minimum resistance of a 1-tree among all 1-trees is 2 . Hence, the stochastic potential of $\left(s_{1}, s_{1}\right)$ is 2 . Similarly, by constructing 2 -trees and 3 -trees we can calculate the stochastic potential of $\left(s_{2}, s_{2}\right)$ and $\left(s_{3}, s_{3}\right)$. The stochastic potential of $\left(s_{2}, s_{2}\right)$ and $\left(s_{3}, s_{3}\right)$ is also 2. Hence, all the Nash equilibria are stochastically stable from Theorem 3.2 . For the case where there is no restriction in coalition formation, CBR dynamics selects $\left(s_{3}, s_{3}\right)$ because it is a strong Nash equilibrium.

\subsubsection{Application to network formation games}

Network formation games are nowadays a consolidated branch of game theory [5, 6, 13]. A network formation game can be viewed as the link formation game given in [7. A pairwise stable network [17] is induced by a strategy profile which is a $\mathcal{K}$-stable equilibrium, where $\mathcal{K}$ is a set of all single player coalitions and all the coalitions of size 2, in the link formation game. In general, the networks which are stable against the deviations of all the coalitions are called 
strongly stable networks. In the literature, there are two definitions of strongly stable networks. The first definition is due to Dutta and Mutuswami [7] in which a strongly stable network is induced by a strategy profile that is an SNE in the link formation game. The second definition is due to Jackson and van den Nouweland [14] in which a strongly stable network is induced by a strategy profile that is an SSNE in the link formation game. Under the stochastic dynamics given by Jackson and Watts [16] a pairwise stable or a network belonging to a closed cycle is a stochastically stable network if its stochastic potential is minimum among all the networks. This result follows from Theorem 3.5 if $\mathcal{K}$ is a set of all single player coalitions and all the coalitions of size 2. It follows from Corollary 3.4 and Corollary 3.6 that under stochastic CBR dynamics all strongly stable networks and closed cycles of networks are stochastically stable.

\section{Conclusions}

We introduce coalition formation among players in an $n$-player strategic game over infinite horizon and propose a CBR dynamics. The mutations are present in the dynamics due to erroneous decisions taken by the coalitions. We show that all $\mathcal{K}$-stable equilibria and all action profiles from closed cycles, that have minimum stochastic potential, are stochastically stable. Similar development holds for strict $\mathcal{K}$-stable equilibrium. When there is no restriction on coalition formation, all SNE and closed cycles are stochastically stable. Similar development holds for SSNE.

\section{Acknowledgements}

This research was sponsored by the European Commission within the framework of the CONGAS project FP7-ICT-2011-8-317672.

\section{References}

[1] R. J. Aumann. Acceptable points in general cooperative n-person games. In R. D. Luce and A. W. Tucker, editors, Contribution to the theory of game IV, Annals of Mathematical Study 40, pages 287-324. Princeton University Press, 1959.

[2] K. Avrachenkov, J. Filar, and M. Haviv. Singular perturbations of markov chains and decision processes. In Handbook of Markov Decision Processes, International Series in Operations Research and Management Science. Springer, 2002.

[3] K. Avrachenkov, J. Filar, and P. Howlett. Analytic perturbation theory and its applications. SIAM, 2013.

[4] K. Avrachenkov, G. Neglia, and V. V. Singh. Network formation games with teams. Technical report, INRIA, https://hal.inria.fr/hal-01097318v2/document, 2014.

[5] G. Demange and M. Wooders, editors. Group Formation in Economics: Networks, Clubs, and Coalitions. Cambridge University Press, 2005.

[6] B. Dutta and M. O. Jackson, editors. Networks and Groups: Models of Strategic Formation. Springer Berlin Heidelberg, 2003.

[7] B. Dutta and S. Mutuswami. Stable networks. Journal of Economic Theory, 76:322-344, 1997. 
[8] D. Foster and H. P. Young. Stochastic evolutionary game dynamics. Theoretical Population Biology, 38(2):219-232, 1990.

[9] D. Fudenberg and L. A. Imhof. Imitation processes with small mutations. Journal of Economic Theory, 131:251-262, 2006.

[10] D. Fudenberg, M. A. Nowak, C. Taylor, and L. A. Imhof. Evolutionary game dynamics in finite populations with strong selection and weak mutation. Theoretical Population Biology, 70:352-363, 2006.

[11] D. Gale and L. S. Shapley. College admissions and the stability of marriage. The Americal Mathematical Monthly, 69(1):9-15, 1962.

[12] J. C. Harsanyi and R. Selten. A general theory of equilibrium selection in games. MIT Press, 1988.

[13] M. O. Jackson. Social and Economic Networks. Princeton University Press, 2010.

[14] M. O. Jackson and A. van den Nouweland. Strongly stable networks. Games and Economic Behavior, 51(2):420-444, 2005.

[15] M. O. Jackson and A. Watts. The evolution of social and economic networks. Journal of Economic Theory, 106(2):265-295, 2002.

[16] M. O. Jackson and A. Watts. On the formation of interaction networks in social coordination games. Games and Economic Behavior, 41:265-291, 2002.

[17] M. O. Jackson and A. Wolinsky. A strategic model of social and economic networks. Journal of Economic Theory, 71(1):44-74, 1996.

[18] M. Kandori, G. J. Mailath, and R. Rob. Learning, mutation, and long run equilibria in games. Econometrica, 61(1):29-56, 1993.

[19] J. G. Kemeny and J. L. Snel. Finite Markov Chains. Springer-Verlag, 1976.

[20] B. Klaus, F. Klijn, and M. Walzl. Stochastic stability for roommate markets. Journal of Economic Theory, 145(6):2218-2240, 2010.

[21] J. Newton. Coalitional stochastic stability. Games and Economic Behavior, 75(2):842-854, 2012 .

[22] J. Newton. Recontracting and stochastic stability in cooperative games. Journal of Economic Theory, 147:364-381, 2012.

[23] J. Newton and S. D. Angus. Coalitions, tipping points and the speed of evolution. Journal of Economic Theory, 157:172-187, 2015.

[24] J. Newton and R. Sawa. A one-shot deviation principle for stability in matching problems. Journal of Economic Theory, 157:1-27, 2015.

[25] A. Roth and M. Sotomayor. Two-Sided Matching: A Study in Game-Theoretic Modeling and Analysis. Cambridge University Press, 1992.

[26] R. Sawa. Coalitional stochastic stability in games, networks and markets. Games and Economic Behavior, 88:90-111, 2014.

[27] H. P. Young. The evolution of conventions. Econometrica, 61(1):57-84, 1993. 